A N N A L E S Annales de Bretagne et des Pays de l'Ouest

\title{
La Grande Brenne, histoire d'une zone humide continentale et de ses étangs
}

Jean-Claude Meuret

\section{(2) OpenEdition}

9 Journals

Édition électronique

URL : https://journals.openedition.org/abpo/4162

DOI : $10.4000 /$ abpo.4162

ISBN : 978-2-7535-7720-6

ISSN : 2108-6443

Éditeur

Presses universitaires de Rennes

Édition imprimée

Date de publication : 18 décembre 2018

Pagination : 170-173

ISBN : 978-2-7535-7718-3

ISSN : 0399-0826

\section{Référence électronique}

Jean-Claude Meuret, "La Grande Brenne, histoire d'une zone humide continentale et de ses étangs »,

Annales de Bretagne et des Pays de l'Ouest [En ligne], 125-4 | 2018, mis en ligne le 18 décembre 2018, consulté le 28 février 2022. URL : http://journals.openedition.org/abpo/4162 ; DOI : https://doi.org/ 10.4000/abpo.4162 
Jean-Yves Éveillard et Jean-Paul Éludut livrent, p. 156-177, les résultats d'une enquête sur " La voie antique de Carhaix (Finistère) à Locmariaquer (Morbihan). Étude de son tracé et réflexions sur son origine et sa fonction ". Cette contribution est la première étude exhaustive sur cette voie dont l'existence s'était perdue au fil du temps. Elle n'a en effet été signalée pour la première fois qu'en 2006. Pour en retrouver le tracé, les deux chercheurs ont mobilisé une grande diversité des sources et de techniques (photographie aérienne, cadastres, photographies du terrain anciennes ou contemporaines, prospection pédestre, lecture des cartes topographiques) et déroulent avec efficacité et bonheur une enquête passionnante. L'article est abondamment illustré.

Comme les éditeurs nous y ont habitué depuis une dizaine d'années, la revue comporte un index des noms de lieux, p. 179-181, une mise en page soignée et une illustration d'une très grande qualité. En une décennie, Aremorica, est désormais bien assise dans le paysage scientifique armoricain et montre le dynamisme de l'archéologie. Les agglomérations, les espaces ruraux, les voies, le monde des morts sont des thèmes récurrents. Aux données du mobilier archéologique s'ajoutent au fil des rares découvertes ou des réinterprétations grâce aux données archéologiques, celles des inscriptions. Un regard rétrospectif montre aussi la complémentarité des techniques et des spécialités, heureusement comprise comme telle par les jeunes chercheurs dont on voit apparaître les noms à côté ou aux côtés de chercheurs confirmés de l'espace géographique concerné par la revue. Cela laisse optimiste sur son avenir et celui de la recherche.

Nicolas MATHIEU

BenARrous, Renaud, La Grande Brenne, histoire d'une zone humide continentale et de ses étangs, 2017, publication du Parc naturel de la Brenne, $66^{\mathrm{e}}$ supplément à la Revue Archéologique du Centre de la France.

Dans ce volume de 437 pages, Renaud Benarrous publie sa thèse soutenue à Paris I Panthéon Sorbonne en 2009, sous la direction de Joëlle Burnouf, augmentée et remaniée dans le cadre de son activité de chargé d'étude au Parc naturel régional de la Brenne (Indre). L'ensemble s'organise en cinq parties. La première énonce les problématiques et les méthodes en 25 pages, la suivante décrit les paysages en 41 pages, une troisième traite de l'espace piscicole lors des périodes préindustrielles en 171 pages, une quatrième est consacrée aux données archéologiques, textuelles et palynologiques en 72 pages, et la dernière tente de formuler la dynamique des objets étudiés dans le temps long en 35 pages.

De la première partie, on retient d'abord un exposé approfondi des études antérieures sur la Brenne, suivi du constat d'une approche historienne du XIX siècle souvent "misérabiliste ", répétant le mythe de la forêt primaire sacrifiée par les déboisements et la métallurgie, puis remplacée par un pays d'étangs grâce à l'action des seuls moines. Quant à sa méthode, l'auteur fait le choix de conjuguer le plus grand nombre de données et pour cela de mettre en ouvre des sources souvent par trop cloisonnées : celles des archives bien sûr, mais aussi celles de la prospection archéologique pédestre et aérienne, ainsi que celles de la palynologie et de la dendrochronologie. On ne peut que louer une telle approche, particulièrement adaptée à l'étude du paysage. Elle multiplie les sources, oblige à les confronter et à les nuancer dans des échelles de temps et d'espace très différentes, et peut par là conduire vers une vision historique moins erronée, plus riche et plus nuancée. Toutefois, on constate vite qu'elle induit 
une somme de travail d'enquête beaucoup plus importante que dans le cadre d'une seule discipline. C'est pour cette raison que l'auteur a choisi de ne traiter que d'un espace assez restreint mais qui constitue le cœur du Parc de la Brenne, celui qu'il nomme la Grande Brenne, et qui ne s'étend que sur 52000 ha.

Dans la deuxième partie, R. Benarrous analyse d'abord les regards portés successivement et aujourd'hui encore sur la Brenne, ce qu'il nomme une " territorialisation mentale " : image d'un pays mauvais car marécageux, humide, malsain, marginal et hors du progrès agraire, volonté d'assainissement scientiste au XIX ${ }^{\mathrm{e}}$ siècle, fermeture et la déprise agraire au xxe siècle au profit de la chasse, à quoi s'ajoute une culture historique réduite à quelques mythes tel celui de Dagobert chassant en Brenne - ceci sur la seule base d'un acte faux - ou des Cisterciens créateurs de la plupart des étangs. Avec un regard d'aujourd'hui, il décrit ensuite ce que l'on nomme un espace piscicole, caractérisé par une très grande densité d'étangs, tous d'apparence anthropique, et en forte croissance à la fin du xxe siècle, mais d'une faible production piscicole par rapport à l'Europe centrale, produisant uniquement des carpes. Sous le regard contemporain enfin, la Brenne bénéficie maintenant d'une représentation et d'un statut à la fois culturels et politiques, tant au niveau de la France que de l'Europe, car elle a été érigée en zone humide à protéger pour sa valeur écologique et sa biodiversité.

C'est en un chapitre beaucoup plus long et touffu qu'il est traité de l'espace piscicole avant l'industrialisation. Après de bonnes descriptions techniques de l'étang, de la chaussée et de sa bonde, R. Benarrous relate tous les types de prospections auxquels il s'est livré pour établir l'inventaire des étangs : représentations cartographiques, cadastre napoléonien, recherches en archives, prospections pédestres à la recherche des chaussées d'étangs abandonnés. Suit une étude du vocabulaire et des noms des étangs, le repérage de chaînes et de réseaux cohérents, leurs implantations, très souvent en milieu et haut de versant, plutôt sur des sols " pauvres ", et leur fonction presque toujours piscicole, mais quasiment jamais attachée aux moulins, sauf dans quelques rares cas de fond de vallée.

Un sous-chapitre aborde ensuite l'épineux problème de leur datation. C'est à juste raison l'occasion de rappeler que les sources écrites ne disent pas tout et qu'elles induisent nombre de biais en raison de leurs lacunes, de leurs origines sélectives, du fort accroissement de la pratique de l'écrit à compter du Xve siècle. À les suivre, le premier moulin de Brenne ne daterait que de 1275 ! À juste titre, l'auteur a mis en œuvre d'autres processus de datation : prospection des étangs asséchés où peuvent être détectés des restes de sites antiques et/ou métallurgiques révélant des sites antérieurs à la mise en eau, datations ${ }^{14} \mathrm{C}$ et dendrochronologiques plus fines, parfois plus anciennes (tableau 28).

Vient ensuite la recherche des propriétaires des étangs pré-industriels. Elle est d'abord l'occasion de rappeler les coutumes anciennes dont on sait la variabilité selon les provinces, mais qui en Touraine comme dans le Maine et l'Anjou, faisaient du droit d'étang un privilège exclusivement seigneurial. De même, selon la tradition historienne, le monde religieux aurait constitué la plus grande partie des détenteurs d'étangs. Retournant aux sources et se livrant au dépouillement de nombreuses archives, l'auteur en vient cependant à nuancer fortement cette règle et cette tradition. Il montre qu'à la fin du Moyen Âge et au XvI ${ }^{\mathrm{e}}$ siècle, 47 \% seulement des sites relevaient de religieux et $53 \%$ de laïques. De plus, ces derniers, n'appartenaient pas seulement au monde seigneurial, mais parfois aussi à celui des bourgeois, des marchands, et même des paysans, mais jamais à des collectivités d'habitants. On ajoutera que, par nature, les archives biaisent les conclusions chiffrées de ce type car elles portent avant tout sur les mondes seigneuriaux ecclésiastiques et laïques, et qu'elles n'émanent que rarement de la sphère roturière. Moins positive, la fin de 
ce chapitre tente d'aborder les cas de conflits entre propriétaires, ou au contraire, d'éventuels programmes d'aménagements cohérents sur un même bassin. Travail sans résultat, et que l'on pourrait juger inutile, elle a cependant le mérite de montrer que toute recherche approfondie nécessite d'examiner le maximum de pistes, fussent-elles des impasses.

En venant aux aspects les plus matériels de la gestion des étangs, et se fondant de nouveau sur les ressources des archives et sur celles de l'archéologie, R. Benarrous présente ensuite la construction des chaussées menée par ceux que l'on nommait les " bessons ". Il détaille tout autant la gestion des plans d'eau, les pêches, puis les modes de commercialisation, toutes activités qui ont privilégié, avant toute autre espèce, la pisciculture de la carpe. Comparée à la plus grande partie du reste de la France, la Grande Brenne présente une très forte spécificité avec seulement neuf moulins et deux hauts fourneaux sur 681 plans d'eau recensés.

Menée à partir d'approches différentes mais complémentaires, cette troisième partie, se termine par un intéressant cahier de documents et de cartes de synthèse. Riche et construite avec logique, elle fournit une bonne mise en perspective des données médiévales et pré-industrielles, et elle amène à dater la majorité des étangs des temps postérieurs au début du XIV ${ }^{\mathrm{e}}$ siècle, laissant cependant dans l'ombre la question de l'étang au Moyen-Âge central.

La quatrième grande partie traite de la Grande Brenne avant le XIV ${ }^{e}$ siècle. En raison de la couverture hydraulique et arborée du territoire, ainsi que de l'absence de fouilles, les données archéologiques de la Pré- et de la Protohistoire sont quasiment absentes. On en connaît quelques-unes pour l'Antiquité, parfois associées à des indices de métallurgie du fer, mais ensuite, et comme souvent, celles du haut Moyen Âge demeurent imperceptibles. Il en est de même pour les sources écrites. Rarissimes, elles ne fournissent rien quant à l'Antiquité, sinon l'appartenance probable du territoire à celui des Bituriges, puis du diocèse de Bourges. Quant à celles du haut Moyen Âge et pour ce qui touche à l'évolution du paysage, elles se résument aux hagiographies ou à un faux diplôme de Dagobert en Brenne, ce qui ne constitue pas les sources les plus fiables. Abordant pour la même période le thème du réseau viaire antique, qui a suscité tant de fausses pistes chez les archéo-historiens du $\mathrm{XIX}^{\mathrm{e}}$ siècle, en Brenne comme ailleurs, R. Benarrous réfute de manière argumentée celle de chaussées d'étangs remployant des tronçons de voies romaines. En revanche, on peut regretter la maigreur des recherches et des informations qu'il produit pour les chemins du second Moyen Âge, hormis le cas d'un chemin dit "des Moines". On sait pourtant que la mise en place des centres puis des territoires paroissiaux, et plus encore celle des châteaux majeurs et des seigneuries moyennes, ont généré nombre de captures viaires. De même, on peut regretter qu'aucune page ne soit consacrée à la fonction viaire des chaussées d'étangs, même si elle peut être demeurée mineure en raison d'une fonction avant tout piscicole.

Pour cette même période pré-piscicole, une grande place est accordée à la réduction directe du fer en bas fourneaux. Elle commence par une étude microtoponymique poussée et se développe surtout à partir des prospections. Celles-ci ont permis de localiser un grand nombre de sites de réduction, parmi lesquels plusieurs ont livré des indices gallo-romains; pour cette période métallurgique, l'auteur emploie même la formule de " caractère industriel ". Il nous semble néanmoins qu'en l'absence de fouilles et de datations sûres, le Moyen Âge peut avoir eu lui aussi sa part dans cette activité.

R. Benarrous consacre enfin un dernier chapitre au rapport entre sociétés prépiscicoles et paysage végétal. S'appuyant de nouveau sur les données écrites, il relève l'apparente multiplication des essartages, telle celle que révèle la disparition de la forêt de Brenne, l'augmentation des mentions d'" effes ", des prairies humides, 
à compter du XIVe siècle, qu'il rapproche de celle des étangs à la même époque. Quant à la palynologie, elle lui fournit des indices indiscutables des occupations humaines jusqu'à l'Antiquité tardive, et peut-être jusqu'au haut Moyen Âge, sur lesquelles il s'appuie à juste titre pour écarter le mythe d'une Brenne humide et marécageuse depuis les origines, et minorer l'importance du rôle des moines dans les défrichements.

Une cinquième et dernière partie reprend les données énoncées dans les précédentes et se donne pour but d'établir le fil de la dynamique qu'a connue la Grande Brenne tout au long des périodes étudiées. Il nous semble qu'au-delà des mots et des théories, elle ne fait le plus souvent que répéter les observations, les explications et les idées déjà exposées. Était-elle vraiment nécessaire, a-t-elle été plaquée et n'auraitelle pas pu être condensée dans la conclusion qui clôt l'ouvrage, laquelle reprend une fois encore l'essentiel de chacune des quatre premières parties?

Il n'est pas simple de formuler un jugement d'historien sur un travail d'une telle importance. Pour ce qui est de la forme du discours, on peut regretter trois choix relatifs au plan de l'ouvrage. En premier lieu, dans une optique de clarté, était-il judicieux de le diviser en autant de parties, d'autant que la dernière aurait pu tenir lieu de conclusion? Ensuite, le choix de traiter en troisième partie d'une période postérieure à celle abordée dans la suivante pourrait se comprendre dans un récit de la recherche menée par l'auteur, mais pour le cas présent, ce n'est pas la finalité majeure de l'ouvrage. Cela instille un peu de confusion dans un discours fourni et riche qui aurait nécessité, au contraire, un ordre plus rigoureux. Enfin, on ne peut manquer de relever le grave déséquilibre entre les différentes parties : la troisième compte à elle seule 171 pages, presque autant que la somme des quatre autres qui n'en totalisent que 173. De ces chapitres trop nombreux, et trop peu équilibrés, il résulte un certain nombre de redondances ainsi que des perturbations dans le fil du discours.

Toutefois, ces manques, peuvent avoir été favorisés par la difficulté à mettre en œuvre une méthode de travail qui, elle, ne peut qu'être louée. Celle-ci a consisté à conjuguer de multiples approches historiques telles les archives, la prospection archéologique, la palynologie, la dendrochronologie, et à les mettre en œuvre dans le temps long. Le projet, qui a nécessité beaucoup de travail et d'assimilation de méthodes bien distinctes, peut paraître ambitieux parce qu'il va à l'encontre de cloisonnements de nos jours de plus en plus marqués. Le premier de ceux-ci remonte à des siècles. Il a posé que l'Histoire ne peut avoir pour fondement que les sources écrites et qu'avant celles-ci il n'est pas d'histoire. Le suivant, plus récent, est né de la création d'une " sous histoire " appelée archéologie, considérée comme " science annexe ", longtemps reléguée aux seuls siècles d'avant l'écriture, avant qu'elle ne conquière son autonomie. À cela s'ajoute le fait que l'Histoire et l'Archéologie, de plus en plus approfondies et institutionnalisées, n'ont cessé de se scinder en périodes et en thèmes de plus en plus étroits et spécialisés. Il en résulte un enrichissement indiscutable des méthodes et des résultats mais aussi une inadéquation totale avec l'étude de certains systèmes complexes et évoluant à un pas séculaire. Or, c'est exactement le cas de la Grande Brenne.

Enfin, le travail de R. Benarrous vaut aussi tout simplement par la richesse de sa matière, par la révision de certains clichés éculés mais tenaces, tel l'immutabilité de la nature, qu'elle soit forêt, zone humide ou étang, par ses découvertes in situ, par ses remises en perspectives. Comme il le conclut lui-même, p. 372, il propose un regard actuel sur " une zone humide " mais appelle aussi les chercheurs spécialistes à approfondir son approche et à la mettre à l'épreuve, en confrontant ses hypothèses aux données "d'autres régions d'étangs et de pisciculture ".

Jean-Claude MEuRET 
Meyer, Christian, Collections de Bretagne, du Centre et des Pays-de-la-Loire. Angers, Blois, Bourges, Chartres, Le Mans, Loches, Nantes, Orléans, Rennes, Tours, Vendôme, Turnhout, Brepols, 2017, xxI-331 p. (Catalogue des manuscrits notés du Moyen Âge conservés dans les bibliothèques publiques de France, 5)

Ce cinquième volume poursuit la collection commencée par le même auteur en 2006, avec un premier volume consacré à la bibliothèque municipale de Colmar [Collections d'Alsace, de Franche-Comté et de Lorraine. I. Colmar, Bibliothèque municipale]. Comme on l'indique dans l'introduction à la collection, " Ce catalogue a pour objet le recensement et la description des manuscrits du Moyen Âge, partiellement ou intégralement notés, ainsi que des fragments ou collections de fragments portant des notations musicales conservés dans les bibliothèques publiques de France. Il est conçu et organisé comme un outil d'investigation pour les historiens, liturgistes, philologues, musicologues ou historiens de l'art du domaine médiéval ".

Le travail de recensement effectué par Christian Meyer est conduit avec extrême rigueur, grâce aussi à la précieuse collaboration de Laura Albiero (Institut de Recherche et d'Histoire des Textes), de Christelle Cazaux-Kowalski (Université de Poitiers) et de Dominique Gatté (Strasbourg). Le référencement bibliographique est enrichi d'une section dédiée aux bases de données en ligne (BVMM, Cantus, DIAMM, etc.), démontrant ainsi une intelligente ouverture aux nouvelles technologies. L'organisation interne des notices, malgré l'absence d'un guide pour le lecteur (un renvoi à un lien internet permet d'accéder à un document pdf avec les Directives pour l'analyse et la rédaction des notices du Catalogue des Manuscrits Notés des bibliothèques publiques de France), est assez intuitive et de facile consultation (les manuscrits sont regroupés par lieu de conservation, et ensuite présentés selon l'ordre de la cote).

La section des index, indispensable outil pour la recherche à l'intérieur d'un catalogue, se limite à l'index analytique des chants, articulé en cinq parties. I. Antiennes et répons, chacune et chacun précédé(e), le cas échéant, par l'identifiant à quatre chiffres de la base Cantus (et non six, comme indiqué dans l'introduction de l'index, étant donné que le double zéro de l'identifiant originaire a été supprimé); six sont, par contre, les chiffres de l'identifiant Cantus Index qui figure, le cas échéant, entre parenthèse à la suite du titre de l'antienne ou du répons. Rien n'est à signaler pour les quatre parties restantes de l'index (II. Hymnes. III. Chants du Propre de la messe. IV. Prose, prosules, éléments de tropes. V. Varia). Bien qu'une partie de l'introduction (p. XV-XVI) fasse déjà le point sur les notations musicales des manuscrits présents dans le catalogue, un index spécifiquement consacré à cet aspect aurait permis une consultation plus systématique. La décision de renoncer à l'index cumulatif des noms de personnes, de lieux, d'institutions et de matières, ainsi qu'à l'index des circonstances liturgiques, pourtant présents dans les autres catalogues de la collection CMN, frustre les attentes d'une recherche plus complexe et ponctuelle.

Quant à l'introduction, la description des dépôts est très détaillée et historiquement documentée. Cependant l'auteur semble parfois sous-estimer les limitations implicites au travail de recensement effectué, comme par exemple celle qui dérive du fait que seulement les bibliothèques publiques ont été dépouillées. En vertu de cette limitation, qui voient exclus les fonds conservés dans les archives départementales et municipales (pour rester dans le domaine public), mais aussi et surtout les fonds des archives et des bibliothèques diocésaines (pour aller dans le privé), chaque tentative de faire le point sur les manuscrits notés ayant survécu des principaux centres religieux français (cathédrales, séminaires ou abbayes) risque à ce stade-là d'apparaître un exercice de style trop audacieux. 
Jones, Michael, Charon, Philippe (dir.), Comptes du duché de Bretagne. Les comptes, inventaires et exécution des testaments ducaux, 1262-1352, Rennes, PUR/Société d'histoire et d'archéologie de Bretagne, coll. "Sources médiévales de l'histoire de Bretagne ", 2017, 512 p. Préface d'Yves Coativy.

L'excellente collection des Sources médiévales de l'histoire de Bretagne repose sur un audacieux postulat : la connaissance de la Bretagne médiévale, pour la période antérieure à la guerre de Succession de Bretagne (1341-1364), se heurte moins à une absence dramatique de documents historiques qu'à leur difficulté d'appréhension, leur éparpillement et leur caractère lacunaire. C'est ce que démontre fort à propos ce beau volume, qui vient enrichir une collection de publications déjà substantielles (Cartulaire de Saint-Guénolé de Landévennec, Cartulaire de Saint-Melaine de Rennes, entre autres).

Le point de départ est fourni par un constat réaliste : contrairement aux chroniques et autres sources narratives, les comptes publics ont longtemps été négligés par les historiens français. Non pas que leur étude ait été inexistante : la Bourgogne, par exemple, a fourni d'extraordinaires séries aux spécialistes des finances ducales. Mais par comparaison, les comptes royaux ou ceux des autres principautés françaises sont des parents pauvres. Dans le cas breton, le problème a été aggravé par les publications quelquefois fautives d'abrégés de comptes édités par les Mauristes, dom Lobineau puis dom Morice. Il a fallu le goût pour les " monuments » de l'histoire de Bretagne, à la fin du XIx ${ }^{\mathrm{e}}$ siècle (La Borderie), puis, après la Deuxième Guerre mondiale, les études plus érudites de Barthélemy-Amédée Pocquet du Haut-Jussé et Yves Renaudin ("Les domaines des ducs de Bretagne. Leur administration du $\mathrm{XIII}^{\mathrm{e}}$ au XVe siècle ", thèse inédite de l'École des Chartes, 1957), pour que ces documents soient pleinement mis en lumière, avant que Jean Kerhervé, dans sa thèse magistrale, n'en tire la grande étude d'histoire sociale, politique et financière qui manquait.

Dans le présent ouvrage, Michael Jones et Philippe Charon, déjà à pied d'œuvre pour l'édition dans la même collection de la thèse de $\mathrm{H}$. Guillotel consacrée aux Actes des ducs de Bretagne (944-1148), se concentrent sur le matériau des comptes, inventaires de succession et exécutions de testaments bretons étendus de 1262 à 1352, qu'ils éditent dans leur intégralité et dans le respect des préconisations de l'École des Chartes. Ces documents se répartissent en neuf sections : abrégés de comptes ducaux (1262-1275); recettes de l'exécution du testament de Jean I ${ }^{\text {er }}$ (12871291); créances et recettes dues à Jean II (1300-1303); inventaire des monnaies et objets précieux déposés dans La Tour neuve de Nantes (1303); dépenses de l'hôtel de Jean II (1305); exécution testamentaire de Jean II (1305-1323); inventaire des monnaies trouvées au trésor de la cathédrale de Nantes après la mort de Jean III (1341); comptes de Pierre Molin le jeune, receveur dans la vicomté de Limoges (1344-1347); comptes d'Alain de Guillemot, châtelain de Touffou (1348-1352). Environ la moitié de ce corpus conservé majoritairement sur des rouleaux de parchemin est inédite. L'ensemble est complété par un glossaire, deux index (noms des lieux et des personnes d'un côté, matières de l'autre), un répertoire des sources et une bibliographie, de même que par un jeu de huit cartes et seize planches en couleur, précédés par une riche introduction de Michael Jones.

Il ressort que la Bretagne, malgré les " effondrements documentaires " de la guerre de Succession du duché, des guerres de Religion ou de la Révolution française, a bien participé, avec un léger retard, et selon une évolution plus simple, de la centralisation et de la sophistication croissantes des pratiques comptables repérables par ailleurs en France durant la "révolution de l'écrit " du XIII et du siècle suivant, mais que cette " bureaucratisation " émergente est difficile à suivre 
en raison des lacunes documentaires. En particulier, l'inspiration de modèles existants, vraisemblablement pris du côté de l'Échiquier de Westminster dans le cas du plus vieil abrégé de comptes ducaux connus (1262), ou de celui des institutions capétiennes, montre un souci de technicité comparable à ce qui se retrouve dans les grandes cours européennes. L'essor d'un milieu de gens de finances ("gens de nos comptes ", selon un document des environs de 1300) est aussi perceptible, par étoffement des rangs des quelques clercs que l'on croise au XIII ${ }^{\mathrm{e}}$ siècle. Le remarquable dossier inédit de l'exécution testamentaire de Jean II, mortellement blessé à Lyon par l'écroulement accidentel d'un mur lors du couronnement du pape Clément V (novembre 1305), révèle la complexité de la tâche en raison de la nature des largesses opérées par le défunt (1000 § aux frères des Carmes de Ploërmel qui accueillent sa sépulture, 6000 \& aux pauvres de Bretagne!), de la longueur des procédures (1305-1323), de la pluralité des intervenants et du nombre des déplacements inhérents. Ce dossier fourmille d'indications rarement connues pour la Bretagne : sur le coût des funérailles ducales (pièce xxxII), celui de l'exécution du tombeau de Jean II à Ploërmel (pièce XXXVI). Il est l'occasion de publier un état descriptif saisissant des aumônes distribuées en deniers, tuniques et paires de souliers aux pauvres du seul diocèse de Rennes, paroisse par paroisse (p. 298-303). Au passage apparaissent les richesses accumulées à la Tour du château de Suscinio, ou à la Tour neuve du château de Nantes, de même que les noms des receveurs et trésoriers qui les manipulent. Ainsi, on peut suivre à la trace ce Rolland Le Lombard, un habile Italien qui s'attache à la cour ducale au point d'en obtenir vraisemblablement la première commission connue en matière de gestion comptable, et qui prospère sous les ducs de la Maison de Dreux en compagnie d'autres officiers de finance d'origine bretonne.

Au total, la moisson est prolifique : histoire financière, institutionnelle, sociale, politique, économique, religieuse, culturelle, histoire locale... Des pans entiers de l'histoire bretonne ressortent mieux éclairés du maniement de cette édition majeure. Nul doute qu'elle en appellera d'autres (il reste beaucoup à faire sur les comptes ducaux des XIV et XV siècles et sur les comptes seigneuriaux). D'ores et déjà, l'on ne peut que remercier les éditeurs pour les avancées que représente ce travail où l'érudition la plus sûre croise la meilleure mise en perspective historique.

Amaury CHAUOU

GuILlET, Bertrand, ARMiDE, Aurélien (dir.), Le château des ducs de Bretagne entre grandeur et renouveau. Huit siècles d'histoire, Nantes, Éditions du château des ducs de Bretagne/Rennes, PUR, 2016, 624 p.

L'édition de beaux livres sur Nantes a le vent en poupe! Après les magnifiques ouvrages publiés en 2013, l'un à Strasbourg consacré à la cathédrale, l'autre à Rennes et évoquant rien moins que la ville, au sens très large, voici " la première monographie du château des ducs de Bretagne " (p. 12). Cette affirmation n'a rien de présomptueux, non pas que les travaux sur cet illustre monument soient rares ou anciens, mais il fallait absolument les amplifier en présentant les recherches archéologiques et historiques récentes, ainsi que les travaux de restauration. Le choix d'un grand format permet d'offrir au lecteur, ravi, de somptueuses illustrations, souvent en pleine page, tant de documents d'archives, de représentations graphiques que de photographies anciennes ou modernes. Beau livre ne signifie pas ici vacuité du discours : si l'objet final peut agréablement adorner un salon où il serait innocemment 